\section{Estudo de Adesão ao Tratamento do Hipotiroidismo}

\section{RESUMO}

Para avaliarmos o impacto da adesão no controle do hipotiroidismo em nosso meio, estudamos 100 portadores de hipotiroidismo mal controlados (Mo de TSH: 12U/L) acompanhados no HC-UNICAMP e no HUCFFUFRJ, 80 mulheres e 20 homens de nivel sócio-econômico e cultural similar. Após serem examinados e orientados quanto à importância de seu uso adequado, os pacientes recebiam hormônio tiroidiano em uma das 4 apresentações comercialmente disponiveis em nosso meio, de forma aleatória e em dose similar à anteriormente prescrita, por um período médio de 3 meses. Os 25 pacientes de cada grupo possuíam características clínicas e sócio-econômico-culturais semelhantes com renda per capita mensal em torno de U\$100,00 ( $X^{2}$ : NS). Mensalmente os doentes eram examinados por um mesmo médico. Realizávamos um controle do número de comprimidos restantes e embalagens vazias, dosagens de TSH e de T4 livre. Nossos resultados mostram que $82 \%$ dos pacientes não aderiam ao tratamento proposto, faltando aos retornos (36\% dos casos) e/ou não seguindo corretamente a prescrição médica (66\% dos casos), Os niveis de TSH foram controlados em 77 pacientes ao término do estudo, independente da formulação de tiroxina prescrita ( $X^{2}$ de pacientes controlados/grupo: NS). Dezesseis pacientes apresentaram niveis de TSH diminuídos no 10 retorno, sugerindo que usavam doses inferiores às prescritas antes do medicamento ser-lhes fornecido. Concluímos que podemos controlar portadores de hipotiroidismo adequadamente com qualquer uma das apresentações disponiveis no mercado nacional quando o paciente adere ao esquema terapêutico proposto. (Arq Bras Endocrinol Metab 2000; 44/6: 483-7)

Unitermos: Adesão; Tratamento; Levotiroxina; Hipotiroidismo.

\section{artigo original}

\author{
Ronnie M. Bagattoli \\ Mario Vaisman \\ Jaderson S. Lima \\ Laura S. Ward
}

\begin{abstract}
Departamento de Clínica Médica, Faculdade de Ciências Médicas da UNICAMP, Campinas, SP $e$ Disciplina de Endocrinologia, HUCFF/UFRJ, Rio de Janeiro, $R J$
\end{abstract}

In order to assess the impact of adherence in the treatment of hypothyroidism, we examined 100 outpatients, 80 women and 20 men from HC-UNICAMP and HUCFF-UFRJ. All had a poor control (Mo TSH: $12 \mathrm{U} / \mathrm{L}$ ). After an initial medical exam and orientation, they randomly received either one of the 4 commercially available preparations of levothyroxine in the same dose previously prescribed, composing 4 groups of 25 patients each. Groups were similar regarding clinic, socioeconomic and cultural profiles, with a monthly income around US\$100.00 per capita ( $X^{2}$ : NS). During a 3 month follow-up they were examined by the same physician and requested to bring all remaining pills and empty packs. Pills were counted and TSH and fT4 were measured monthly. We found $82 \%$ of non-compliance either with visit attendance ( $36 \%$ of the patients) or with the correct prescription ( $66 \%$ of the patients). At the end of the study 77 patients presented normal TSH levels independently of the levothyroxine preparation administered ( $X^{2}$ controlled patients/group: NS). TSH levels dropped below normal range in 16 patients at their 1st return suggesting that they were previously using lower doses than the pre-

Recebido em $10 / 02 / 00$ Revisado em 18/04/00 e em 23/05/00 Aceito em 29/05/00 
scribed one. We conclude that hypothyroidism may be adequately treated with any of the commercially available formulations provided the patient complies with therapy. (Arq Bras Endocrinol Metab 2000; 44/6: 483-7)

Keywords: Adherence; Treatment; Levothyroxine; Hypothyroidism.

B AIXA ADESÃo Ao TRATAMENTO contribui de forma importante para falha terapêutica, principalmente nas doenças infecto-contagiosas e nas doenças crônicas (1-5). Também é a principal causa de níveis séricos elevados de TSH em pacientes tomando doses inapropriadas de levotiroxina para seu tamanho ou que apresentam grandes variações nos testes de função tiroidiana com a mesma dose (6). No hipotiroidismo congênito, a baixa adesào ao tratamento pode significar perdas irreversíveis para o sistema nervoso central, assim como danos no crescimento e desenvolvimento normal (7). Para o indivíduo adulto, o tratamento inadequado também pode ter conseqüências sérias na performance cardíaca, no perfil lipídico, em vários aspectos neurocognitivos e até mesmo estar associado com depressão e outros transtornos afetivos (8-11). $\grave{A}$ primeira vista, nada poderia ser mais simples do que tratar o hipotiroidismo. Afinal, possuímos ensaios muito sensíveis de TSH, que permitem diagnosticar $\mathrm{e}$ acompanhar com precisão a resposta à terapêutica (12). Possuímos levotiroxina (L-T4) em diferentes apresentações e suficientes dosagens para adequar a terapia de reposição aos requerimentos individuais. $\mathrm{O}$ medicamento possui um custo relativamente baixo. No entanto, muitos de nossos pacientes mantêm nívcis elevados de TSH, mesmo em hipotiroidismo sintomático.

Para avaliarmos a importância da adesão ao tratamento na prática clínica de serviços públicos, onde a maior parte de nossa população é atendida, conduzimos um estudo paralelo de adesão ao tratamento nos Ambulatórios de Medicina Interna da FCM-UNICAMP e de Endocrinologia do HUCFF da UFRJ.

\section{MATERIAL E MÉTODOS}

Foram estudados 100 pacientes portadores de hipotiroidismo mal controlado acompanhados no Ambulatório Geral de Adultos do Hospital de Clínicas da Faculdade de Ciências Médicas da Universidade Estadual de Campinas/UNICAMP e no Ambulatório de Endocrinologia do Hospital Universitário Clemente Fraga Filho da Universidade Federal do Rio de Janeiro/UFRJ, 80 mulheres e 20 homens de nível sócio econômico similar (renda média per capita em torno de 2 salários mínimos, ou cerca de US $\$ 100,00$ ). A idade dos pacientes variou de 18 a 78 anos $(M o=56$ anos). Dados sobre a renda dos pacientes, condições de alimentação e moradia e características sócio-culturais foram obtidos através de entrevistas com pesquisadoras do Serviço Social e Nutrição.

Todos tinham diagnóstico confirmado de hipotiroidismo há cerca de 2 anos ( $\mathrm{Mo}=2$ anos) e, apesar de já haverem sido consultados várias vezes desde o seu diagnóstico ( $\mathrm{Mo}=4$ retornos), mantinham níveis de TSH elevados (entre 7 e $50 \mathrm{U} / \mathrm{L}, \mathrm{Mo}=$ $12 \mathrm{U} / \mathrm{L}$ ). Em $46 \%$ dos casos tratava-se de portadores de tiroidite de Hashimoto, 38 pacientes haviam sido submetidos a procedimentos ablativos, sendo 16 destes por doença de Basedow-Graves e o restante não possuía etiologia definida. Nenhum paciente possuía evidência de qualquer causa de má absorção ou tomava qualquer medicamento passível de interferir com a absorção ou ação da levotiroxina.

Quatro grupos de 25 pacientes receberam o hormônio tiroidiano $\mathrm{em}$ uma das 4 apresentações comercialmente disponíveis em nosso meio: Puran $\mathrm{T}^{\circledR}{ }^{\circledR}$; Euthyrox ${ }^{\mathbb{R}}$; Tetroid $^{\circledR}$ e Synthroid ${ }^{\circledR}$. As drogas foram fornecidas pelos respectivos laboratórios especificamcnte para o estudo (as três primeiras) ou sob a forma de amostras grátis (Synthroid ${ }^{\mathbb{B}}$ ). A apresentação de levotiroxina designada a cada paciente foi determinada de forma randomizada previamente. Após serem examinados, os pacientes eram cuidadosamente orientados quanto à importância do uso contínuo e adequado da medicação prescrita, sua dose, horário e condições ideais de tomada. A dose para cada paciente foi mantida dentro de uma faixa de 1,7 a $2,0 \mu \mathrm{g} / \mathrm{Kg}$ de peso, conservando-se a dose que vinha sendo anteriormente prescrita.

Os doentes eram mensalmente examinados pelo mesmo médico, recendo uma quantidade de comprimidos pouco maior do que a suficiente até o retorno, durante um período de 3 meses. Eram orientados a retornar as embalagens vazias a cada consulta assim como os comprimidos sobressalentes. Realizávamos uma contagem mensal do número de comprimidos restantes e embalagens vazias assim como dosagens de TSH e de T4 livre.

O TSH foi dosado através de método fluoroimunoenzimático - Stratus II System da Baxter Diagnostics Inc., IL-USA. O valor mínimo detectado, segundo o fabricante do kit, é de $0,05 \mathrm{U} / \mathrm{L}$ e os valores normais variam entre 0,38 e $6,15 \mathrm{U} / \mathrm{L}$; coeficiente de variação intra e inter-ensaio de $3,5 \%$ e $10,1 \%$ para va- 
lores baixos, 3,2\% e 5,7\% para médios e 3,0\% e 5,1\% para elevados.

Os níveis de T4 livre foram dosados também por método fluoroimunoenzimático - Stratus II System. A sensibilidade funcional do kit é de $0,2 \mathrm{ng} / \mathrm{dL}$ e os valores normais variam entre $0,74 \mathrm{e} 2, \mathrm{lng} / \mathrm{dL}$.

Para descrever o perfil da amostra foram feitas tabelas de frequiência das variáveis categóricas e estatísticas descritivas das variáveis contínuas. Para verificar a diferença de distribuição de freqüência das var:̈̌veis categóricas entre os 4 grupos, foram feitas tabelas de contingência $\mathrm{c}$ utilizados os testes de associação do Qui-quadrado $\left(\mathrm{X}^{2}\right)$ ou, quando necessário, o teste exato de Fisher. Foram consideradas significativas as diferenças cujos testes tiveram $\mathrm{p}$ com valor menor que $5 \%$, ou seja, $\mathrm{p}<0,05$.

\section{RESULTADOS}

A renda mensal média foi de cerca de $\mathrm{R} \$ 200,00$ (1 salário mínimo e meio ou cerca de US $\$ 100,00$ ) per capita $\mathrm{em} 82 \%$ dos casos; 2 salários mínimos $\mathrm{em} 9 \% \mathrm{e}$ em apenas outros $9 \%$ dos pacientes é superior a essa quantia. Os 4 grupos cram similares em relação à idade, sexo e condição sócio-econômica dos pacientes $\left(\mathrm{X}^{2} ; \mathrm{p}=\mathrm{NS}\right)$.

Nossos resultados mostram que $82 \%$ dos pacientes não seguiam adequadamente a orientação médica faltando aos retornos (36\% dos casos) e/ou não seguindo a prescrição médica ( $66 \%$ dos casos). As causas alegadas para a não obediência às recomendações médicas foram "puro esquecimento" em $67 \%$ dos casos, falta de entendimento adequado sobre o uso contínuo c permanente da medicação em $28 \%$ dos pacientes e intolerância à droga em 3 casos. Destes, 1

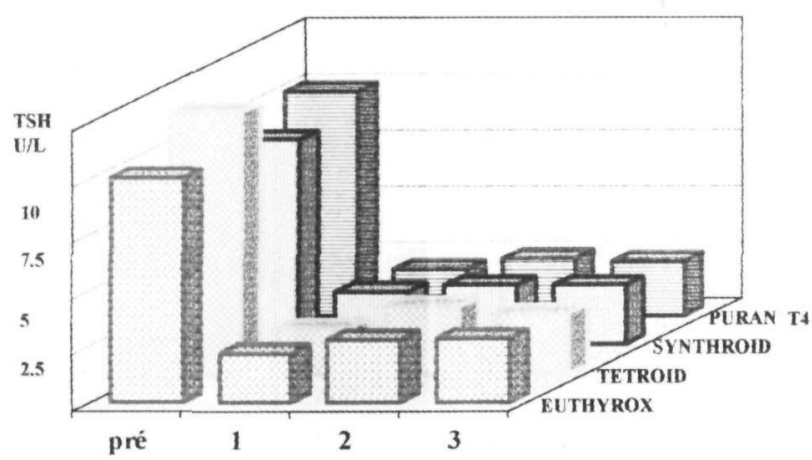

Figura 1. Evolução dos niveis médios de TSH nos pacientes usando levotiroxina sob as 4 apresentações comerciais disponiveis em nosso meio: Puran T4(B); Synthroid( $($ ), Tetroid( $(B)$ e Euthyroxß. Estão representados os niveis médios de TSH em $U / L$ encontrados pré-intervenção e na $1^{a}, 2^{\circ}$ e $3^{g}$ consultas de retorno. paciente se queixou de angina pectoris e os outros 2 de palpitações. Dois pacientes simplesmente não retornaram ao ambulatório.

Na figura 1 representamos a evolução dos níveis de TSH médios nas 3 consultas. Dezesseis pacientes apresentaram, no primeiro retorno, níveis de TSH abaixo dos limites de normalidade, sugerindo que a dose que afirmavam tomar anteriormente era maior do que a que necessitavam. Nenhum destes pacientes suspendeu a medicação ou apresentou queixa sugestivas de excesso de dosagem. Em 77 pacientes os níveis de TSH foram controlados até o término do estudo, independente da formulação de levotiroxina prescrita. Os 23 pacientes restantes permaneciam com níveis de TSH acima da normalidade ao término dos 3 meses de estudo. Não observamos diferença na distribuição de pacientes controlados ou não controlados entre os 4 grupos terapêuticos ( $\mathrm{X}^{2}$ de pacientes controlados/ grupo= NS). Também não observamos diferenças clínicas ou na velocidade de compensação entre os 4 grupos terapêuticos.

\section{DISCUSSĀO}

O hipotiroidismo é uma condição comum na população com prevalência aproximada de $2 \%$ das mulheres adultas e $0,2 \%$ dos homens adultos (12). Em indivíduos acima de 60 anos, sua prevalência atinge mais de $10 \%$ das mulheres e de 2 a $4 \%$ dos homens (13). A doença ilustra bem as dificuldades relacionadas a adesão a tratamento de doenças crônicas. Apesar do tratamento ser relativamente simples, pois envolve basicamente terapia substitutiva com hormônio tiroidiano, de fácil seguimento graças aos recursos laboratoriais amplamente disponíveis e de custo relativamente baixo, o indivíduo com hipotiroidismo é, freqüentemente, considerado um paciente "difícil". Isso se deve a uma série de motivos. O principal é o fator econômico-cultural. Apesar do custo mensal do tratamento variar entre US\$2 e 8 , ele ainda é alto para uma significativa parcela da população cujo nível de renda nào passa dos US $\$ 100,00$ mensais. Vale lembrar que $16 \%$ da população da região sudeste, a mais desenvolvida do Brasil, apresenta estado de pobreza, definido pelo SUS como menos de $1 / 2$ salário mínimo de renda per capita (14).

O hipotiroidismo, não raramente está associado a várias co-morbidades, o que além de aumentar o número de medicamentos que o paciente tem de aplicar ou ingerir todo dia, também aumenta o peso desses medicamentos em seu orçamento. Entretanto, o fator econômico foi diminuído cm nosso estudo pelo 
fornecimento gratuito da levotiroxina. Existem ainda fatores decorrentes da própria doença, como o estado de letargia, de desânimo e o esquecimento cuja instalação insidiosa muitas vezes não é notada pelo paciente ou por seus familiares, nem lembrada pelo médico. Outro fator é a perenidade do tratamento. Nem todos os pacientes compreendem ou assumem bem o fato de que o tratamento deve ser vitalício e terào de conviver com a necessidade de tomar medicamento todo dia pelo resto de suas vidas. A melhora da compreensão sobre sua patologia, a cducação dos pacientes e sua crença na efetividade do tratamento melhoram sua adesão à tcrapêutica, isso não apenas em hipotiroidismo $(3,15,16)$.

Programas institucionais de registro dos doentes com convocação automática têm se mostrado eficientes e benéficos em termos de custo-efetividade (17). Estudos em populações de nível sócio-econômico-cultural bem mais elevado que a nossa mostram níveis de TSH anormais em $48 \%$ dos usuários de levotiroxina (concentrações de TSH elevadas em $27 \% \mathrm{e}$ diminuídas em $21 \%$ dos casos) (18). Devemos lembrar que quase $10 \%$ da população do sudeste do Brasil tem menos de 1 ano de estudo e $26 \%$ menos de 3 anos de estudo (19). A presença de efeitos colaterais também contribui para a falta de adesão. Em nossa casuística, $3 \%$ dos pacientes se queixaram de sintomas possivelmente devidos a superdosagem. Um número significativo de doentes (16\%) acabou recebendo uma dose maior do que a necessária. É provável que estes pacientes não tivessem sintomas anteriores simplesmente porque não seguiam a recomendação médica. Quando insistimos para que tomasse a dose prescrita e esta lhes foi fornecida, estes pacientes devem ter recebido uma dose excessiva de tiroxina. A criação de programas institucionais baseados nos níveis de TSH poderia diminuir a baixa adesão e os problemas decorrentes de dosagem inadequada (20).

Dados de Werner e col. de 1988 mostravam variação no conteúdo de $\mathrm{L}-\mathrm{T} 4$ em preparados comerciais disponíveis $\mathrm{cm}$ nosso meio, levando a uma preocupação para com a formulação prescrita (2l). No entanto, não observamos diferenças clínicas no uso de qualquer dos preparados disponíveis no nosso meio, ratificando dados de literatura que mostram a bioequivalência das levotiroxinas no tratamento do hipotiroidismo $(22,23)$. Nossos dados mostram que, à semelhança do relatado em outras doenças crônicas, os portadores de hipotiroidismo são freqüentemente mal controlados por falta de adesão ao tratamento proposto $(3,24,25)$.

Concluímos que podemos controlar adequadamente pacientes com hipotiroidismo com qualquer uma das apresentaçòes disponíveis no mercado nacional, desde que o paciente adira ao esquema terapêutico proposto. Constância no tratamento, observação da dose e horário adequado de tomada são fundamentais para garantir o sucesso terapêutico do hipotiroidismo. Antes de modificar a dose ou a apresentação, devemos assegurar-nos de que o paciente está realmente tomando diariamente a dose prescrita, na forma recomendada. Nossa experiência, à semelhança do recomendado na literatura, sugere que a melhor maneira de melhorar a adesão ao tratamento consiste na sua simplificação, relacionando o horário da tomada com as atividades do doente, em particular o horário da $l^{a}$ refeição diária e o uso de um único comprimido na dosagem adequada $(6,26)$. Tão importante quanto essas medidas, se faz conscientizar o paciente do caráter permanente do tratamento e incentivá-lo a acreditar que cle pode proporcionar considerável melhora $\mathrm{em}$ sua qualidade de vida.

\section{AGRADECIMENTOS}

Os autores agradecem às empresas SANOFI, MERCK e ACHÉ o gentil oferecimento de medicamentos para todo o protocolo de estudo, e à KNOLL pelo oferecimento de amostras grátis. Agradecemos ainda à Dra. Beatriz C. Quinet Leimann pelo esquema de randomização de tratamentos.

\section{REFERÊNCIAS}

1. Iseman MD. Treatment and implications of multidrugresistant tuberculosis for the 21 st century. Chemotherapy 1999:45:34-40

2. Melfi CA, Chawla AJ, Croghan TW. Hanna MP, Kennedy $S$. Sredl K. The effects of adherence to antidepressant treatment guidelines on relapse and recurrence of depression. Arch Gen Psychiatry 1998;55: 1128-32.

3. Garay Sevilla ME, Malacara HJM, González Parada F, Jordán Ginés $L$. The belief in conventional medicine and adherence to treatment in non-insulin-dependent diabetes mellitus patients. J Diabet Complicat 1998; 12:239-45.

4. Mehta SS, Wilcox CS, Schulman KA. Treatment of hypertension in patients with comorbidities: results from the study of hypertensive prescribing practices (SHyPP). Am J Hypertens 1999: 12:333-40

5. Morris AD, Boyle DIR, McMahon AD, Greene AS, MacDonald TM, Newton RW. Adherence to insulin treatment glycaemic control, and ketoacidosis in insulin-dependent diabetes mellitus. Lancet 1997:350:1505-10.

6. Hanna FWF, Lazarus JH, Scanlon MF. Controversial aspects of thyroid disease. BMJ 1999:319:894-9.

7. Harrell GB, Murray PD. Diagnosis and management of congenital hypothyroidism. J Perinat Neonatal Nurs 1998; 11:75-85. 
8. González Vílchez F, Castillo L, Pi J, Ruiz E. Cardiac manifestations of primary hypothyroidism. Determinant factors and treatment response. Rev Esp Cardiol 1998:51:893-900

9. Martinez Triguero ML, Hernandez Mijares A, Nguyen T, Muñoz ML, Peña H. Morillas C, et al. Effect of thyroid hormone replacement on lipoprotein (a), lipids, and apolipoproteins in subjects with hypothyroidism. Mayo Clin Proc 1998; 73:837-41.

10. Dugbartey AT. Neurocognitive aspects of hypothyroidism. Arch Intern Med 1998;158:1413-8.

11. Sullivan GM, Hatterer JA, Herbert J, Chen X, Roose SP, Attia $E$, et al. Low levels of transthyretin in the CSF of depressed patients. Am J Psych 1999: 156:710-5.

12. Sawin $C$, Castelli WP, Hershman JM, MCNamara $P$, Bacharach $P$. The aging Thyroid. Thyroid deficiency in the Framingham Study. Arch Intern Med 1985; $145: 1386-8$

13. Parle JV, Ranklyn JA, Cross KW, Jones SC, Sheppard MV. Prevalence and follow up of abnormal thyrotrophin (TSH) concentrations in elderly in the United Kingdom. Clin Endocrinol 1991;34:77-83.

14. Indicadores e Dados Básicos Brasil 1998. Ministério da Saúde, Rede Interagencial de Informaçôes para a Saúde; http://datasus.saude gov.br/

15. Wood LC. Support groups for patients with Graves' disease and other thyroid conditions. Endocrinol Metab Clin North Am 1998:27:101-7.

16. Schectman JM, Elinsky EG, Pawlson LG. Effect of education and feedback on thyroid function testing strategies of primary care clinicians. Arch Intern Med 1991;151:2163-6.

17. Davies PDO, Lazarus JH. Wheeler MH. A computer-assisted thyroid follow-up system. World J Surg 1986;10:681-6.

18. Parle JV, Franklyn JA, Cross KW, Jones SR, Sheppard MC. Thyroxine prescription in the community: serum thyroid stimulating hormone level assays as an indicator of undertreatment or overtreatment. Br J Gen Pract 1993:43:107-9.

19. IBGE / Pesquisa Nacional por Amostra de Domicilios -
PNAD. Indicadores socio-econômicos básicos para 1998. Ministério da Saúde, Rede Interagencial de Informações para a Saúde; http://datasus.squde gov.br/

20. Tan SY, Gill GV, Hales T. Thyroid register audit: a district general hospital experience. Postgrad Med J 1992:68:483-4.

21. Werner RS, Romaldini JH, Braverman LE, Management of hypothyroidism: variation in levothyroxine (L-T4) preparations. In: Meirelles RMR, Machado A, Póvoa LC, eds. Clinical Endocrinology. Proceedings of the 18th Brazilian Congress of Endocrinology and Metabolism, Rio de Janeiro, 1988. Amsterdam: Elsevier Science Publishers, 1988: $157-60$.

22. Dong BJ, Hauck WW, Gambertoglio JG, Gee L, White JR, Bubp JL, et al. Bloequivalence of generic and brandname levothyroxine products in the treatment of hypothyroidism. JAMA 1997:277:1205-13.

23. Cerutti R, Rivolta G, Cavalieri L, Di Giulio C, Grossi E, Vago $T$, et al. Bioequivalence of levothyroxine tablets administered to a target population in steady state. Pharmacol Res 1999:39:193-201.

24. Davidson BL. A controlled comparison of directly observed therapy vs. self-administered therapy for active tuberculosis in the urban United States. Chest 1998: 1 14:1239-43.

25. Pierin AMG, Mion Jr D. Adesão ao tratamento antihipertensivo. Conceito, avaliação e fatores determinantes. Hipertensäo 1999:2:9-12.

26. Ain KB, Pucino F, Csako G, Wesley RA, Drass JA, Clark C, et al. Effects of restricting levothyroxine dosage strength availability. Pharmacotherapy 1996:16:1103-10.

\section{Endereço para correspondência:}

Laura Sterian Ward

Cidade Universitária Zeferino $\mathrm{Vaz}$

13.083-970 Campinas, SP

Tel/Fax: (55) (19) 289-4107

e.mail:ward@unicamp.br 